\title{
Redes sociales, herramientas de comunicación masiva para cuidado y preservación de los recursos naturales a través de difusión de prácticas químicas ambientales y de resultados estadísticos en los hogares
}

Social networks, mass communication tools for care and preservation of natural resources through diffusion of environmental chemical practices in homes

Susana Monserrat Zurita Polo. ${ }^{1}$, Iraida Maritza Gavilánez Álvarez. ${ }^{2}$ \& Marco Velasco Arellano. $^{3}$

\begin{abstract}
.
DOI: https://doi.org/10.33262/cienciadigital.v3i3.1.674 Ecuador has a mega diversity of natural resources: renewable as plants, animals, water, soil, among others; non-renewable, such as minerals, metals, oil, among others; and inexhaustible in ecological time such as wind, sunlight, energy from the waves of the sea. These are material goods and services provided by nature and are of great importance for the welfare of society, as they provide raw materials, minerals and food for living beings. At present the state of these at world-wide level is worrisome, annually it is diminishing the capacity of the ecosystems to produce goods and services by the overexploitation, misuse and non-preservation of the natural resources on the part of the human being.

The human is the responsible for the deterioration and diminution of the biodiversity and therefore of the ecosystems and natural resources; it is convenient to raise awareness about the importance of such valuable resources, motivate the population to contribute to the care and preservation of resources from their personal, family and social space; It is everyone's responsibility to make this world a better place.
\end{abstract}

\footnotetext{
1 Escuela Superior Politécnica de Chimborazo, Facultad de Recursos Naturales. Riobamba, Ecuador, susana.zurita@espoch.edu.ec

${ }^{2}$ Escuela Superior Politécnica de Chimborazo, Facultad de Recursos Naturales. Riobamba, Ecuador, igavilanez@espoch.edu.ec

3 Escuela Superior Politécnica de Chimborazo, Facultad de Recursos Naturales. Riobamba, Ecuador, jhalmar63@gmail.com
} 
This study promotes the use of social networks as mass communication tools for the care and preservation of natural resources through environmental chemical practices to students of the Faculty of Natural Resources of the Higher Polytechnic School of Chimborazo (ESPOCH) who will be the active spokespersons for the care and diffusion of the importance of the correct handling and disposal of common household chemicals; a sample of 120 students was considered, users of social networks with ages older than 17 years, determining that the most used social networks in this sense are Facebook, YouTube and WhatsApp for the dissemination of information.

Keywords: social networks, natural resources, environmental practices

\section{Resumen.}

Ecuador cuenta con una mega diversidad de recursos naturales: renovables como plantas, animales, agua, suelo, entre otros; no renovables como minerales, metales, petróleo, etc.; e inagotables en el tiempo ecológico como viento, luz solar, energía de las olas del mar. Éstos son bienes materiales y servicios que brinda la naturaleza y son de gran importancia para el bienestar de la sociedad, ya que proveen materia prima, minerales y alimentos para los seres vivos. En la actualidad el estado de éstos a nivel mundial es preocupante, anualmente va decreciendo la capacidad de los ecosistemas de producir bienes y servicios por la sobreexplotación, mal uso y no preservación de los recursos naturales por parte del ser humano.

El ser humano es responsable del deterioro y disminución de la biodiversidad y por ende de los ecosistemas y recursos naturales; es conveniente crear conciencia sobre la importancia de tan valiosos recursos, motivar a la población a aportar al cuidado y preservación de los recursos desde su espacio personal, familiar y social; es responsabilidad de todos, el hacer de este mundo un lugar mejor.

El presente estudio promueve el uso de redes sociales como herramientas de comunicación masiva para el cuidado y preservación de los recursos naturales a través de prácticas químicas ambientales a estudiantes de la Facultad de Recursos Naturales de la Escuela Superior Politécnica de Chimborazo (ESPOCH) quienes serán los portavoces activos para el cuidado y difusión de la importancia del manejo correcto 
y desecho de productos químicos de uso común en los hogares; se consideró una muestra de 120 estudiantes, usuarios de redes sociales con edades mayores a 17 años, determinando que las redes sociales más utilizadas en este sentido son Facebook, YouTube y WhatsApp para la divulgación de información.

Palabras claves: Redes sociales, Recursos Naturales, Prácticas químicas ambientales

\section{Introducción.}

Hoy por hoy el mundo está siendo sacudido por la velocidad para generar, producir e innovar ideas, información, conocimientos y saberes, que, apoyados en la ciencia y tecnología son transmitidos de forma masiva, rápida y global a través de las Tecnologías de la Información y de la Comunicación, las cuales han evolucionado aceleradamente por su capacidad de interconexión a través de la Red; esta nueva fase de desarrollo tiene gran impacto en las organizaciones y en la sociedad a todo nivel, sin dejar de lado las relacionadas con el medio ambiente y los recursos naturales, la adaptación del entorno a este nuevo potencial y la adecuada utilización del mismo supone un reto sin precedentes.

Las innovaciones tecnológicas proporcionan cada vez nuevas y mejores alternativas de comunicación, así como variadas fuentes de información y difusión de estilos de comportamiento, actitudes, valores, formas de organización, etc. en este sentido y dado que la humanidad está inmersa en la llamada sociedad de la información, hay que considerar que los datos son la materia prima del siglo XXI, a mayor información mayor capacidad de acción se tendrá.

La incorporación de las TIC y específicamente de las Redes Sociales como herramientas del mundo físico al mundo digital permite nuevas formas de acceder, generar, y transmitir información y conocimientos, promoviendo el ampliar las perspectivas en este caso del cuidado y preservación del medio ambiente y los recursos naturales renovables, no renovables e inagotables en el tiempo ecológico, desde el ámbito individual y familiar por medio de prácticas ambientales en los hogares; puesto que hábitos cotidianos como arrojar la basura sin separarla, comprar utensilios desechables o adquirir alimentos envasados en materiales antiecológicos o no reciclables contribuyen en gran medida a la contaminación medioambiental y degradación global del planeta. En este sentido una comunicación efectiva con usuarios del entorno cercano y alrededor del mundo, sin limitaciones de espacio ni tiempo, de manera sincrónica y/o asincrónica, por medio de redes sociales de fotografía, de mensajería o de interacción total son un elemento clave para crear conciencia y difundir en la población aspectos relacionados al gran aporte que se puede hacer al planeta por medio de 
prácticas ambientales en el hogar, colegio, universidad o desde cualquier ámbito donde se desarrolle.

Las redes sociales en Internet son comunidades virtuales donde personas, organizaciones, empresas interactúan con usuarios de todo el mundo con quienes encuentran gustos o intereses en común. Funcionan como una plataforma de comunicaciones que permite conectar gente que se conoce o que desea conocerse, y que les permite centralizar recursos, como fotos y vídeos, en un lugar fácil de acceder y administrado por los usuarios mismos. (Castro, 2017)

El cuidado y preservación del medio ambiente, turismo y difusión de los recursos naturales cada vez va tomando mayor impulso y movimiento en las redes sociales, en tal virtud en el presente artículo se tratarán aspectos a tomar en cuenta para elevar las potencialidades del uso de Internet, Redes sociales y plataformas tecnológicas para la preservación y cuidado de los recursos naturales a través de la difusión de buenas prácticas ambientales en el hogar dando a conocer los riesgos que pueden generar afectación a la salud por la presencia de sustancias químicas de uso común presentes en los residuos .

\section{Metodologia.}

El punto de partida para el presente trabajo es el uso de Redes Sociales como herramienta de comunicación directa con los usuarios. Las técnicas utilizadas en el presente estudio son: observación directa, encuestas, entrevistas; las encuestas fueron aplicadas a estudiantes de las Escuelas de Turismo y Recursos Naturales Renovables de la Escuela Superior Politécnica de Chimborazo (ESPOCH) en Riobamba - Ecuador, para identificar si se realiza prácticas ambientales como clasificación y manejo adecuado de residuos, reciclaje, huertos familiares, entre otros, así como el uso que dan a las redes sociales, el contenido que publican y/o comparten en relación al manejo y prácticas químicas ambientales en el hogar en pro del cuidado y preservación del medio ambiente.

\section{Resultados.}

El cuidado y preservación de los Recursos Naturales en nuestro medio es considerado de gran importancia, sin embargo, la sociedad en general espera que las acciones para dicho efecto las realicen las instituciones públicas, gubernamentales o privadas como Ministerio del Ambiente, gobiernos seccionales como GADs provinciales, municipales, parroquiales, etc., sin asumir la responsabilidad de cada individuo; cada persona puede aportar a la conservación de los recursos naturales y medio ambiente en general, desde el ámbito personal, familiar, educativo y social, mediante el uso adecuado de prácticas ambientales que incluyan una correcta clasificación de los residuos del hogar, especialmente de aquellos que generan problemas de contaminación por sus componentes químicos . 
Es importante proyectar al mundo las maravillas naturales que el Ecuador posee y cómo se puede proteger y cuidar los recursos naturales desde cada uno de los hogares; actualmente se cuenta con herramientas que facilitan este proceso de una forma global y masiva llegando a todos los rincones del mundo, cada vez son más las personas que utilizan nuevas tendencias de comunicación a través de estructuras virtuales que permiten la transmisión de información a través del Internet y de manera más específica por medio del uso de las famosas "Redes Sociales".

La comunicación a través del Internet está dando lugar a que cada vez más personas se conviertan en usuarios directos y activos de las Redes Sociales, quienes las utilizan como herramientas atractivas de comunicación y difusión de información para compartir por medio de comentarios, imágenes, mensajes cortos, mensajes relacionados a un mismo contexto mediante la utilización de hashtag "\#” o etiquetas de almohadilla, videos, etc., opiniones y criterios de determinado tema; dejando de lado limitaciones en cuanto a espacio físico y temporal, promoviendo su participación en actividades de apoderamiento de información y conocimientos, contribuyendo a mejorar el ambiente.

La mayoría de productos que se utilizan a diario en el hogar contienen productos químicos que pueden generar afectaciones a la salud pero su presencia es importante, ya que gracias a ellos en el hogar es posible eliminar bacterias, insectos, mantener limpias las superficies, los inodoros, utensilios de cocina, etc., su inadecuado puede generar alergias en la piel, contaminación de los ambientes por la presencia de sustancias volátiles que pueden provocar nauseas, trastornos en la visión, dolores de cabeza, etc., es decir que muchos productos sin siquiera sospecharlo pueden causar daños, por ello es recomendable realizar una buena clasificación de los envases de cada uno de estos productos ya que generalmente los comercializan en envases de plástico.

Los residuos se pueden clasificar de varias maneras, ya sea por las características asociadas al manejo, por el estado físico y por el origen, en el presente trabajo se hará referencia a los residuos generados por las características asociadas a manejo, dentro de los cuales se encuentran los residuos sólidos orgánicos (biodegradables), residuos sólidos inorgánicos (inertes), desechos sólidos generales (general contaminación ambiental), desechos sólidos pétreos (residuos de escombros y demoliciones), desechos peligrosos (peligro para el ambiente y la salud humana). (Gavilanez M. , 2012)

Dentro de los daños que pueden generar estos residuos se puede citar a los detergentes lavaplatos que contienen entre sus componentes químicos fosfatos y cloro que generan contaminación en los mantos acuíferos; insecticidas y raticidas que contienen cianuro, carbonatos, compuestos organofosforados, estricnina inclusive talio que son contaminantes de la capa de ozono; cierto tipo de shampoo que entre sus componentes están el amoniaco, nitratos, naftalina, percloroetileno y fragancias sintéticas que pueden provocar alteraciones a nivel de los riñones e incluso generar daños en la vista; blanquedores que contienen el 
hidróxido de potasio o de sodio, peróxido de hidrogeno, hipoclorito de sodio o de calcio que causan daños permanentes en la piel, además su uso excesivo daña a los suelos en donde son desechados; alcohol medicinal cuyo uso excesivo puede generar problemas en el hígado, náuseas, vómitos, pérdida de conocimiento, además puede ser inflamable por lo que puede causar incendios; maquillaje cuyos componentes son cloruro de polivnilo, lauril, sulfato de sodio, triclosaan, formaldehidos, los cuales pueden causar cáncer, alteración de la fertilidad, obesidad, acné, interfiere con las funciones hormonales y generan contaminación ambiental; pastas dentales que contienen amoniaco, etanol, fluoruros, alcohol, saborizantes, colorante vegetal donde algunos de estos componentes son de origen mineral (abrasivos) que se extraen de la canteras o minas los cuales alteran de manera importante el paisaje y los ecosistemas, envases PET cuyo polímero se obtiene mediante una reacción de policondensación entre el ácido tereftálico y el etilenglicol, su fabricación involucra sustancias toxicas, presencia de metales pesados y químicos irritantes los cuales contaminan el ambiente, sal común o cloruro de sodio, es una sustancia no contaminante pero al contacto con los ojos puede provocar perdida de la visión, adema causa danos a los riñones, limpiadores del horno los cuales contienen en su mayoría lejías que son contaminantes de la capa de ozono. (Chang, 2002)

En cuanto a los hábitos y prácticas de las personas y los hogares respecto a temas ambientales como clasificación de residuos, prácticas de ahorro de agua y energía, transporte y movilidad, pautas de consumo y conciencia ambiental de acuerdo al Módulo de información ambiental en hogares de la encuesta nacional de empleo, desempleo y subempleo (ENEMDU) del Instituto Nacional de Estadísticas y Censos en el año 2017 el 47,7\% de los hogares ecuatorianos realizaron clasificación de los residuos, esto significa que aproximadamente cinco de cada diez hogares realizaron esta práctica, siendo el plástico el residuo más clasificado con el 32,98\%, seguido por residuos orgánicos, papel cartón y vidrio. La provincia que más realiza esta práctica ambiental es Galápagos donde los hogares clasifican sus residuos en un $98,08 \%$. (INEC, 2017)

Con respecto al uso de pilas en el año 2017 los hogares utilizaron pilas recargables en un $8,01 \%$ y pilas no recargables en un $91,99 \%$ lo que equivale a que en los hogares se utilizan más de 14 millones de pilas, de las cuales el 83,97\% terminan con el resto de la basura y apenas el 5,24\% son entregadas a centros de acopio o contenedores especiales, el 5,54\% fueron enterradas o arrojadas a quebradas y el porcentaje restante fueron guardadas, vendidas o regaladas. Similar situación atraviesa los focos ahorradores, una vez utilizados son arrojados con el resto de la basura en un $86,72 \%$, apenas el 3,35\% son depositados en centros de acopio o contenedores especiales y el resto fueron vendidos, regalados, guardados, quemados, enterrados o arrojados a quebradas. (INEC, 2017), lo ideal sería que retornen a sus fabricantes para que les den un uso adecuado reutilizando todo el material que a ellos les resulte útil, ya que estos desperdicios pueden ser tóxicos, corrosivos, inflamables, etc. Las pilas contienen mercurio, zinc, litio, cadmio, carbono y plata que pueden ser precursores de 
cáncer, causar irritaciones en la piel y posible enseñamiento además de contaminación del ambiente (Chang, 2002)

Sobre las acciones pro-ambientales más realizadas en los hogares son: cerrar la llave mientras jabonan los platos, se cepillan los dientes o se bañan $(93,28 \%)$, ducharse en menos de diez minutos $(78,09 \%)$, revisar regularmente las tuberías $(58,62 \%)$, utilizar balde en lugar de manguera para ciertas actividades $(52,92 \%)$ y reusar el agua $(40,22 \%)$.

En el 2017, al menos $80 \%$ de los hogares demuestran realizar cuatro prácticas de ahorro de energía, como son: apagar los focos al salir de una habitación (96,77\%), evitar introducir alimentos calientes en el refrigerador $(93,22 \%)$, abrir cortinas y persianas para aprovechar la luz del sol $(87,73 \%)$ y desconectar los aparatos eléctricos y electrodomésticos cuando no se usan $(79,69 \%)$. (INEC, 2017)

Con estos antecedentes se aplicaron encuestas a 120 estudiantes de la Facultad de Recursos Naturales de la ESPOCH, para identificar las prácticas ambientales que realizan de forma personal o familiar, lastimosamente los resultados obtenidos son aún más bajos a la media nacional. Según los resultados obtenidos el 20,83\% de los estudiantes encuestados realizaron clasificación de los residuos, esto significa que apenas dos de cada diez hogares realizaron esta práctica, siendo las botellas de plástico el residuo más clasificado, seguido por el papel. En cuanto al uso de pilas las personas encuestadas manifestaron que en promedio utilizan 8 pilas por hogar, de las cuales el $25 \%$ corresponde a pilas recargables, pero al terminar su vida útil el $90,90 \%$ de los encuestados manifiestan las arrojan a la basura ordinaria por cuanto desconocen de lugares o centros de acopio para la recolección y tratamiento de las mismas. Similar situación se atraviesa con el uso de focos, una vez utilizados son arrojados con el resto de la basura en un $95,83 \%$, apenas el 4,16\% son reciclados y/o depositados en centros de acopio o contenedores especiales.

Con respecto a las prácticas pro-ambientales realizadas por los encuestados: cerrar la llave mientras jabonan los platos, se cepillan los dientes o se bañan (75\%), ducharse en menos de diez minutos (70,83\%), revisar regularmente las tuberías $(20 \%)$, utilizar balde en lugar de manguera para ciertas actividades (45\%) y reusar el agua (37,5\%). Sobre las prácticas de ahorro de energía, como apagar los focos al salir de una habitación los encuestados manifiestan hacerlo $(79,16 \%)$, evitar introducir alimentos calientes en el refrigerador (95\%), abrir cortinas y persianas para aprovechar la luz del sol (90\%) y desconectar los aparatos eléctricos y electrodomésticos cuando no se usan $(69,17 \%)$.

Con los resultados obtenidos se ha determinado la importancia de concienciar a la sociedad en general sobre la realización de prácticas ambientales en el hogar, lo que sería de gran aporte para la preservación y cuidado del medio ambiente y los recursos naturales, con este propósito se identifica que una opción muy apropiada es la difusión de información por medio de imágenes, videos, estados, comentarios sobre las prácticas ambientales que se 
pueden realizar en el hogar en pro de un mundo mejor a través del uso de Tecnologías de Información y Comunicación, concretamente con el uso de Redes Sociales.

Las redes sociales se han convertido, en pocos años, en un fenómeno global, éstas se expanden como sistemas abiertos en constante construcción de sí mismos, al igual que las personas que las utilizan. Las redes sociales se han universalizado, se las han incorporado plenamente en la vida y se han convertido en un espacio idóneo para intercambio de información y conocimiento de una forma rápida, sencilla y cómoda. (Aguilar, Campos , \& Batlle, 2012)

El término red, proviene del latín rete, hace mención a una estructura que tiene un determinado patrón; por lo que puede aplicarse en diversos ámbitos, como la informática donde una red es un conjunto de equipos interconectados que comparten información. En cuanto al término social, es relativo a la sociedad o conjunto de individuos que interactúan entre sí para formar una comunidad, pudiendo definir las Redes Sociales como estructuras en donde muchas personas mantienen algún tipo de vínculo ya sea amistad, comercial, laboral u otro.

Hoy en día el término "red social " se refiere a los diferentes sitios o páginas de internet que favorecen la creación de comunidades virtuales, ofreciendo a sus usuarios la posibilidad de contactarse con infinidad de individuos a fin de compartir contenidos, interactuar y crear comunidades sobre intereses similares: trabajo, lecturas, juegos, amistad, relaciones amorosas, entre otros. (Zurita \& Monge, 2018)

Las redes sociales en Internet, engloban numerosas y variadas redes, por lo que generalmente, se habla de ellas en plural. Es difícil encontrar una persona que sólo posea una cuenta en uno de los servicios que ofrece Internet. Normalmente, los individuos son usuarios activos de varios servicios, mediante los cuales, crean y mantienen diferentes relaciones personales. Los servicios de redes sociales se están convirtiendo en un agregado de muchos servicios que funcionaban de forma separada. Se puede trasladar un estado de Twitter a Tuenti, o ver un vídeo en Youtube y compartirlo en Facebook o un estado en Instagram o Whatsapp. Todo esto integra a los usuarios de un modo complejo, por lo que el concepto de servicio de red social se diluye, y acaba siendo una combinación de posibilidades de comunicación.

Las redes sociales son precisamente una de las formas que internet brinda de comunicación con las personas. Se enmarcan en la denominación de Web 2.0., y suponen una forma de interacción social que se basa en el intercambio interactivo y dinámico de distinta información entre diferentes personas, grupos o instituciones. (Muñoz, Sandra, \& Barreiro, 2013)

Las prácticas sociales se establecen mediante las actividades propuestas por el servicio que, habitualmente, son: Compartir contenido como fotografías, vídeos, páginas web, textos, mensajes cortos, música o noticias, enviar mensajes privados "chatear" con otros usuarios, 
participar en juegos sociales que ofrece el servicio, comentar el contenido compartido por otros usuarios, publicar eventos para anunciar acontecimientos a su red de contactos, hablar en tiempo real con uno o más usuarios mediante chat o sistemas de conversión grupal, crear grupos exclusivos para determinados contactos, publicar comentarios en el perfil o espacio personal de otros usuarios. Para el cuidado y preservación de los recursos naturales, y la difusión de prácticas ambientales se puede hacer uso de redes sociales para lo cual se debe considerar varios aspectos como: la conversación es la base de una relación interpersonal, Twitter para hablar al minuto, Facebook para hablar cada día, Google Plus para hablar a la semana, Linkedin para hablar con profesionales, entre otros; la seguridad en la red depende del sentido común de cada usuario, el factor humano es de gran importancia en Internet. (Zurita \& Monge, 2018)

En Ecuador, según la Encuesta de condiciones de vida realizada por el Instituto Nacional de Estadísticas y Censos (INEC, 2018), 4’224.984 habitantes que representan el 25,28\% de la población del Ecuador utilizan redes sociales, considerando que Facebook es la red social de mayor acogida, es pertinente indicar que el mayor porcentaje de usuarios en Facebook tiene edad entre 18 y 25 años, seguido muy de cerca por los de 26 a 35 años. En Ecuador existen 13,5 millones promedio de usuarios de internet; a julio de 2018: 12 millones son usuarios de Facebook, 3,8 millones de Instagram, 0,8 millones activos de Twitter y 6,1 millones de Whatsapp. (Del Alcazar, 2018)

Con estos antecedentes es importante dar a conocer las prácticas ambientales que se pueden promover a través de las plataformas digitales y que se pueden poner en práctica desde el hogar, ambiente educativo y personal, como:

- Clasificación de los residuos, separando adecuadamente considerando si son: plástico, papel, residuos orgánicos, material que puede ser reutilizado y/o reciclado, etc.

- Uso mínimo de pilas o a su vez preferir utilizar pilas recargables, una vez terminada su vida útil, dar el tratamiento adecuado y no arrojar a la basura ordinaria.

- Uso adecuado de focos y lámparas fluorescentes, se sugiere utilizar focos ahorradores, una vez utilizados no deben ser arrojados con el resto de la basura de ser posibles promover su reutilización, caso contrario deben ser depositados en centros de acopio o contenedores especiales.

Es importante determinar que el sistema de manejo de residuos sólidos se compone de cinco sub sistemas que son: generación, recolección y transporte, reciclaje, tratamiento y disposición final. (Gavilanez \& Zurita, 2019)

Las medidas preventivas para la generación de desechos consisten en la implementación de las 3R, es decir, reducir, reusar, reciclar en la medida de lo posible. Para ello se debe implementar el sistema de separación de desechos y de compactación del cartón, papel y plástico para disminuir su volumen, para de esta manera reducir al máximo los desechos y optimizar la disponibilidad de espacio en el centro de acopio. (Muñoz P. , 2008) 
Con respecto a las prácticas pro-ambientales:

- Cerrar la llave mientras jabonan los platos, se cepillan los dientes o se bañan.

- Ducharse en menos de diez minutos, procurar que el calor no se excesivo

- Revisar regularmente las tuberías

- Utilizar baldes en lugar de manguera

- Reusar el agua de ser posible

Sobre las prácticas de ahorro de energía:

- Apagar los focos al salir de una habitación

- Evitar introducir alimentos calientes en el refrigerador

- Abrir cortinas y persianas para aprovechar la luz solar

- Desconectar los aparatos eléctricos y electrodomésticos cuando no se usan

Variables consideradas

El cuestionario fue realizado considerando aspectos básicos relacionados a: prácticas ambientales en el hogar y uso de redes sociales: Tipo de Redes sociales, tiempo empleado para publicaciones, contenido y elementos a difundir por medio de las redes sociales, medios digitales utilizados para la comunicación; obteniendo los siguientes resultados:

Tabla 1. Redes sociales utilizadas

\begin{tabular}{lc}
\hline \multicolumn{1}{c}{ Red Social } & Porcentaje \\
\hline Facebook & $80 \%$ \\
Whatsapp & $85 \%$ \\
Twiter & $23 \%$ \\
Instagram & $45 \%$ \\
Snapchat & $10 \%$ \\
Google + & $15 \%$ \\
Youtube & $63 \%$ \\
Otras & $20 \%$ \\
\hline
\end{tabular}

Fuente: Elaboración propia

En cuanto a la red social más utilizada las personas encuestadas manifiestan que es Whatsapp, seguida por Facebook, por la facilidad de comunicación a través de teléfonos inteligentes preferentemente.

Tabla 2. Tiempo empleado para uso de Redes sociales

\begin{tabular}{lc}
\hline \multicolumn{1}{c}{ Uso de Red Social } & Porcentaje \\
\hline Diariamente & $55 \%$ \\
Semanalmente & $30 \%$ \\
\hline
\end{tabular}


Vol. 3, N³.1, p. 29-44, julio - septiembre, 2019

\begin{tabular}{lc}
\hline \multicolumn{1}{c}{ Uso de Red Social } & Porcentaje \\
\hline Mensualmente & $10 \%$ \\
Ocasionalmente & $5 \%$
\end{tabular}

Fuente: Elaboración propia

De acuerdo a los encuestados el uso de redes sociales en su mayoría se lo realiza diariamente, incluso manifiestan en muchos de los casos que el número de horas diarias destinadas para el efecto sobrepasan las 3 horas.

Tabla 3. Interacción y contenidos compartidos en Redes sociales

\begin{tabular}{lc}
\hline \multicolumn{1}{c}{ Contenido en Red Social } & Porcentaje \\
\hline Académico & $15 \%$ \\
Social & $76 \%$ \\
Profesional & $10 \%$ \\
Negocios & $35 \%$ \\
Turismo & $30 \%$ \\
Cuidado y preservación de & $25 \%$ \\
recursos naturales & $15 \%$ \\
\hline
\end{tabular}

Fuente: Elaboración propia

En cuanto a la interacción y al tipo de contenido compartido en redes sociales, se relaciona mayoritariamente al ámbito social, los contenidos no siempre son validados y la información no necesariamente es verificada y/o contrastada, por tal razón puede promover que se visualice información falsa o no adecuada.

Tabla 4. Contenidos compartidos en Redes sociales relacionados a prácticas ambientales

\begin{tabular}{lc}
\hline \multicolumn{1}{c}{ Contenido en Red Social } & Porcentaje \\
\hline Cuidado y uso adecuado del agua & $65 \%$ \\
Cuidado del suelo, formas de protección & $40 \%$ \\
Como evitar la contaminación del aire & $22,5 \%$ \\
Uso adecuado de energía & $22,5 \%$ \\
Pautas para protección del medio ambiente & $68,33 \%$ \\
Acciones para evitar el uso de material & $62,5 \%$ \\
desechable y plástico & $16,67 \%$ \\
Otros & \\
\hline
\end{tabular}

Fuente: Elaboración propia 
Considerando que la educación ambiental es un proceso que afecta a la persona no solo en la etapa de la Educación formal, sino que tiene una clara inclinación hacia lo actitudinal y comportamental, por cuanto debe basarse en la adquisición de conciencia, conocimiento, actitudes, aptitudes, capacidad de evaluación y participación, se determinó los contenidos que generalmente se comparten en cuanto a prácticas ambientales. (González Muñoz, 1996)

\section{Discusión}

El uso de nuevas tecnologías para difusión y transmisión de información está revolucionando la forma de pensar de los usuarios de las redes sociales, por medio de contenidos se puede alcanzar a usuarios alrededor del mundo y motivar su participación con prácticas ambientales desde el hogar para el cuidado y protección de los recursos naturales; se pretende que cada vez es sea más frecuente y más sencillo obtener y transmitir información que pueda ser utilizada y puesta en práctica en beneficio de la sociedad y de ésta y de futuras generaciones.

Colocar la información en internet tiene como ventaja el acceso a la información desde cualquier ubicación, la información reside en servidores con conexión permanente; las herramientas Web 2.0 proporcionan mejoras en cuanto a interfaz, lo que posibilita una mejor y más fluida comunicación.

Entidades gubernamentales han visto en el uso de Internet una alternativa eficiente para la difusión de proyectos ambientales en Ecuador, como lo hacen el Ministerio del Ambiente y Agua, Ministerio de Turismo, Ministerio de Energía y Recursos Naturales No Renovables entre otros, con el objetivo de dar a conocer a la comunidad información relacionada al medio ambiente, sin embargo se ha identificado que a través de publicaciones compartidas en redes sociales, la información se vuelve masiva y llega a mayor cantidad de usuarios.

En la actualidad plataformas como Youtube, presentan la información de forma gráfica y activa sobre las prácticas ambientales en el hogar, como por ejemplo cómo realizar la clasificación y eliminación de residuos, cómo reciclar, cómo hacer huertos orgánicos, prácticas de ahorro de agua, de energía, conciencia ambiental, etc.

Las redes sociales como Facebook, Whatsapp, Youtube, se han convertido en espacios donde se puede mostrar al mundo las maravillas que ofrece la naturaleza y los recursos que ofrece, y cómo protegerlos y preservarlos, es importante tener información actualizada acerca de temas de interés, además permiten acudir a eventos, participar en actos y conferencias tanto presenciales como virtuales y que pueden ser en tiempo real son ventajas del uso de las Redes sociales para preservación de los Recursos Naturales.

Hay que considerar también que las Redes sociales pueden llegar a ser peligrosas en cuanto a si no se configura la privacidad correctamente, puede haber violación de los derechos de 
autor, así como pueden darse casos de suplantación de identidad, y problemas de contenido mal intencionado y erróneo, usuarios inescrupulosos pueden apoderarse de contenidos publicados y utilizarlos equivocadamente para beneficio propio perjudicando notablemente al autor. Adicionalmente se debe tomar muy en cuenta que comentarios emitidos en redes sociales pueden generar inconvenientes y malos entendidos, razón por la cual es importante hacer buen uso de las mismas.

\section{Conclusiones.}

- Cada vez es más frecuente el uso de internet y Redes Sociales para la difusión de prácticas químicas ambientales en el hogar con el fin de preservar la integridad personal, lo que permite crear conciencia en usuarios y por medio de ellos a sus familias sobre el cuidado y preservación del medio ambiente y los Recursos Naturales.

- La preservación, cuidado del medio ambiente y entorno familiar, pueden ser conocidos y apreciados a nivel mundial a través del Internet, entidades de gobierno como Ministerio del Ambiente y Agua, Ministerio de Turismo, Ministerio de Energía y Recursos Naturales No Renovables, entre otros, pueden hacer uso del Internet a través de las Redes Sociales ya que se han convertido en una herramienta de difusión muy utilizada por entidades públicas y privadas, llegando a los usuarios con mensajes de alerta, disminuyendo los costos de comunicación,

- El uso de redes sociales implica tener conciencia de las ventajas que puede tener en cuanto a publicidad y/o difusión de información y servicios, pero de la misma forma se deberá considerar el tipo de información que se coloca ya que ésta estará al alcance de todo el mundo, por lo que es importante poner atención sobre todo en lo relacionado a seguridad y privacidad.

- El uso de sustancias en el hogar para las múltiples funciones que son creadas debe promover un cuidado adecuado y al momento de desechar los distintos envases considerados como residuos se lo debe hacer de una manera adecuada para evitar la formación de compuestos que provoquen contaminación.

- Es importante motivar a las personas para crear una conciencia ambiental a fin de preservar el medio ambiente y los recursos naturales

\section{Referencias bibliográficas.}

Aguilar, M., Campos , S., \& Batlle, P. (2012). El uso académico de las redes sociales en universitarios. Comunicar, 19, 131-138. Obtenido de http://dx.doi.org/10.3916/C382012-0304\%5Cnhttp://www.revistacomunicar.com/indice/articulo.php?numero=38-2012-16 
BRANDEC. (2018). Hablemos de marcas. Recuperado el 20 de 03 de 2018, de Estadísticas de redes sociales en Ecuador 2017: http://www.hablemosdemarcas.com/estadisticasde-redes-sociales-en-ecuador-2017/

Castro, L. (14 de 2 de 2017). About Español. Recuperado el 13 de 1 de 2019, de ¿Qué es una red social?: https://www.aboutespanol.com/que-es-una-red-social-158168

Chang, R. (2002). Quimica. Mexico: Mc Graw Hill.

Del Alcazar, J. (27 de 01 de 2018). Formación Gerencial. Obtenido de Estado digital Ecuador Julio 2018: http://blog.formaciongerencial.com/ecuador-cifras-digitalesperfil-de-consumidor-y-ranking-sitios-web/

El emprendedor. (2017). Errores al usar Facebook en la empresa. Recuperado el 23 de 03 de 2018, de http://www.elemprendedor.ec/errores-al-usar-facebook-en-la-empresa/

Gavilanez, M. (2012). Plan de Manejo Ambiental en el Botadero de basura del Canton Guamote. Obtenido de http://www.lareferencia.info/vufind/Record/EC_02a174d1542bd0673bb186065442 $313 b$

Gavilanez, M., \& Zurita, M. (enero de 2019). Difusion del manejo de residuos solidos en el restaurante de la escuela de gastronomia de la facultad de salud publica-ESPOCH. Obtenido de https://www.eumed.net/rev/caribe/2019/01/manejo-residuossolidos.html

González Muñoz, C. (1996). Principales tendencias y modelos de la Educación Ambiental en el sistema escolar. Revista Iberoamericana de educación, 13-74. Obtenido de http://www.rieoei.org/oeivirt/rie11.htm

INEC. (2017). Instituto Nacional de Estadísticas y Censos. Obtenido de http://www.ecuadorencifras.gob.ec/documentos/webinec/Encuestas_Ambientales/Hogares/Hogares_2017/DOC_TEC_MOD_AMBIEN TAL_ENEMDU\%202017.pdf

INEC. (2018). ECUADOR EN CIFRAS. Obtenido de Estadísticas Sociales: http://www.ecuadorencifras.gob.ec/documentos/webinec/Estadisticas_Sociales/TIC/2016/170125.Presentacion_Tics_2016.pdf

Muñoz, M., Sandra, M., \& Barreiro , F. (2013). La Importancia De Las Redes Sociales En El Ambito Educativo. EA, Escuela abierta: revista de Investigación Educativa, 91104. Obtenido de Muñoz, M., Sandra, M., \& Barreiro, F. (2013). La Importancia De Las Redes Sociales En El Ambito Educativo. EA, Escuela Abierta: Revista de Investigación Educativa, 16, 91-104. Retrieved from https://s3.amazonaws.com/academia.edu.documents/40756605/1._prin 
Muñoz, P. (2008). Manual de Manejo de Residuos Solidos Urbanos. Quito, Ecuador: Industria Grafica.

Zurita, S., \& Monge, P. (07 de 2018). Eumed. Revista Caribeña. Obtenido de https://www.eumed.net/rev/caribe/2018/07/plataformas-tecnologicasgastronomia.html 


\section{PARA CITAR EL ARTÍCULO INDEXADO.}

Zurita Polo, S., Gavilánez Álvarez, I., \& Velasco Arellano, M. (2019). Redes sociales, herramientas de comunicación masiva para cuidado y preservación de los recursos naturales a través de difusión de prácticas químicas ambientales y de resultados estadísticos en los hogares. Ciencia Digital, 3(3.1), 29-44. https://doi.org/10.33262/cienciadigital.v3i3.1.674

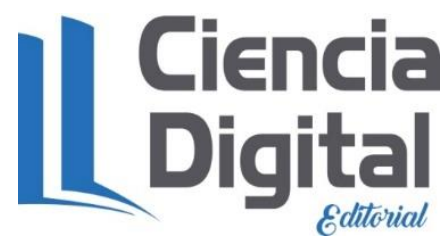

El artículo que se publica es de exclusiva responsabilidad de los autores y no necesariamente reflejan el pensamiento de la Revista Ciencia Digital.

El artículo queda en propiedad de la revista y, por tanto, su publicación parcial y/o total en otro medio tiene que ser autorizado por el director de la Revis
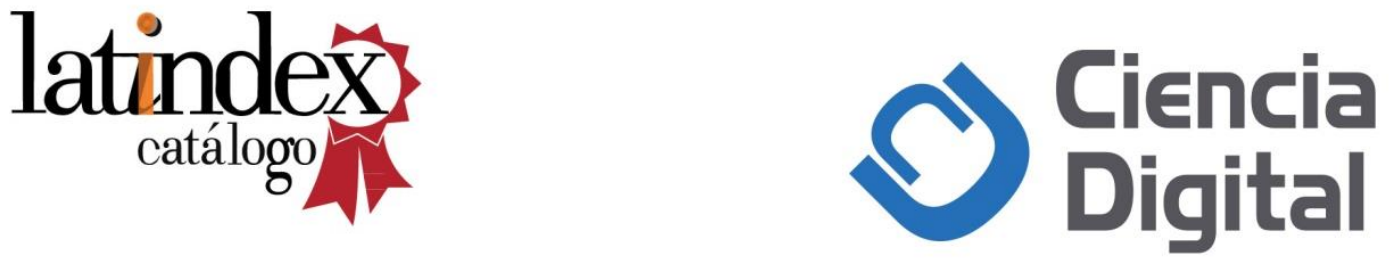